Fixed Point Theory, 19(2018), No. 2, 643-658

DOI: $10.24193 /$ fpt-ro.2018.2.51

http://www.math.ubbcluj.ro/ ${ }_{\text {nodeacj/sfptcj.html }}$

\title{
ON GENERALIZED METRIC SPACES AND GENERALIZED CONVEX CONTRACTIONS
}

\author{
CECE LI* AND DONG ZHANG** \\ *School of Mathematics and Statistics \\ Henan University of Science and Technology \\ Luoyang 471023, People's Republic of China \\ E-mail: ceceli@haust.edu.cn \\ College of Mathemat- ics and System Science, \\ Shandong University of Science and Technology, \\ Qingdao 266590, People's Republic of China \\ ** Department of Mathematics, Peking University \\ Beijing 100871, People's Republic of China \\ E-mail: dongzhang@pku.edu.cn
}

\begin{abstract}
In this paper, we study the generalized metric introduced by Branciari. We find an induced metric of the generalized metric, by which some new properties of the generalized metric are presented. As a main result, we generalize several generalized, unified and extended fixed point theorems on generalized convex contractions.

Key Words and Phrases: Generalized metric, approximate fixed point, generalized convex contraction, $\alpha$-admissible mapping.
\end{abstract}

2010 Mathematics Subject Classification: 47H10.

Acknowledgements. The first author was supported by grant of NSFC-11401173, and the Innovation Team of Henan University of Science and Technology (No.2015XTD010).

\section{REFERENCES}

[1] H. Aydi, E. Karapinar, B. Samet, Fixed points for generalized $(\alpha, \psi)$-contractions on generalized metric spaces, J. Ineq. Appl., 2014(2014), Article ID 229.

[2] H. Aydi, E. Karapinar, D. Zhang, On common fixed points in the context of Branciari metric spaces, Results Math., 71(2017), 73-92.

[3] A. Branciari, A fixed point theorem of Banach-Caccioppoli type on a class of generalized metric spaces, Publ. Math. Debrecen., 57(2000), 31-37.

[4] M. Berinde, Approximate fixed point theorems, Stud. Univ. Babes-Bolyai, Math., 51(2006), no. 1, 11-25.

[5] N. Bilgili, E. Karapınar, A note on "Common fixed points for $(\psi, \alpha, \beta)$-weakly contractive mappings in generalized metric spaces", Fixed Point Theory Appl., 2013(2013), Article ID 287.

[6] N. Hussain, M.A. Kutbi, P. Salimi, Fixed point theory in $\alpha$-complete metric spaces with applications, Abstract and Applied Analysis, 2014(2014), Article ID 280817. 
[7] N. Hussain, E. Karapinar, P. Salimi, F. Akbar, $\alpha$-admissible mappings and related fixed point theorems, J. Ineq. Appl., 2013(2013), Article ID 114.

[8] W.A. Kirk, N. Shahzad, Generalized metrics and Caristi's theorem, Fixed Point Theory Appl., 2013(2013), Article ID 129.

[9] U. Kohlenbach, L. Leustean, The approximate fixed point property in product spaces, Nonlinear Anal., 66(2007), 806-818.

[10] A. Latif, M. Eshaghi Gordji, E. Karapinar, W. Sintunavarat, Fixed point results for generalized $(\alpha, \psi)$-Meir-Keeler contractive mappings and applications, J. Ineq. Appl., 2014(2014), Article ID 68 .

[11] A. Latif, W. Sintunavarat, A. Ninsri, Approximate fixed point theorems for partial generalized convex contraction mappings in $\alpha$-complete metric spaces, Taiwans Journal of Mathematics, 19(2015), no. 1, 315-333.

[12] M.A. Miandaragh, M. Postolache, S. Rezapour, Approximate fixed points of generalized convex contractions, Fixed Point Theory Appl., 2013(2013), Article ID 255.

[13] S. Reich, Approximate selections, best approximations, fixed points, and invariant sets, J. Math. Anal. Appl., 62(1978), 104-113.

[14] B. Samet, C. Vetro, P. Vetro, Fixed point theorems for $\alpha-\psi$-contractive type mappings, Nonlinear Anal., 75(2012), 2154-2165.

[15] W.A. Wilson, On semi-metric spaces, Amer. J. Math., 53(1931), no. 2, 361-373.

Received: March 24, 2016; Accepted: July 28, 2016. 
